\title{
ABIETANES AND KAURANES FROM LEAVES OF CRYPTOMERIA JAPONICA
}

\author{
Wen-Chiung Su, Jim-Min FANG and Yu-Shia Cheng* \\ Department of Chemistry, National Taiwan University, Taipei, Taiwan 10617, Republic of China
}

(Received 10 August 1993)

Key Word Index-Cryptomeria japonica; Taxodiaceae; leaves; diterpenes; abietane-type; kauranetype.

\begin{abstract}
Totarol, 14 abietanes, two secoabietanes and four ent-kaurane derivatives were isolated from the leaves of Cryptomeria japonica. The new compounds included 6,12-dihydroxyabieta-5,8,11,13-tetraen-7-one, 6 $\beta$-hydroxyferruginol, $7 \alpha, 8 \alpha$-epoxy-6 $\alpha$-hydroxyabieta- $9(11), 13$-dien-12-one, $(5 R, 10 S)$-12-methoxyabieta-6,8,11,13-tetraene, ent-kaur-15en-17-al and (+)-16-acetylkaurane-16,17-diol. Their structures were determined by chemical and spectral methods.
\end{abstract}

\section{INTRODUCTION}

The Japanese cedar, Cryptomeria japonica D. Don., is a widely distributed conifer called 'sugi' in Japanese [1]. Its constituents have been extensively studied [2-12]. The leaves are reported to contain diterpenes ferruginol (1), sugiol (5), xanthoperol, kaurane, kaurene and phyllocladene. We report herein novel diterpenes of abietane- and kaurene-types (11-14, 20 and 21) isolated from the ethyl acetate-soluble part of the leaves of $C$. japonica.

\section{RESULTS AND DISCUSSION}

Ten known abietane-type diterpenes, ferruginol (1) [13, 14], 6,7-dehydroferruginol (2) [13], 6,7-dehydroferruginol methyl ether (3) [13], 7-dehydroabietanone (4) [15], sugiol $(5)[14,16], 6 \alpha$-hydroxysugiol (6) [14], hinokiol (7) [17], 11-hydroxysugiol (8) [18], cryptojaponol (9) [19] and 5-epixanthoperol (10) [20], a secoabietane dialdehyde (15), 12-hydroxy-6,7-secoabieta-8,11,13-triene-6,7dial [21] and its methyl ether (16) [21], as well as totarol (17) [22], were identified by comparison of their physical and spectral data (mp, [ $\alpha]$, mass, IR, ${ }^{1} \mathrm{H}$ and ${ }^{13} \mathrm{C}$ NMR) with the literature. The methyl ethers 3 and 16 are reported for the first time from a natural source, although they have been prepared by methylation of 2 and 15, respectively [21]. The C-5 chirality of 16 was confirmed by NOE of the aldehyde signal at $\delta 9.86$ upon irradiation of $\mathrm{Me}-10$ at $\delta 1.49$.

The molecular formula $\mathrm{C}_{20} \mathrm{H}_{26} \mathrm{O}_{3}$ of 11 was deduced from its exact mass $[\mathrm{M}]^{+}$at $\mathrm{m} / \mathrm{z}$ 314.188. The IR absorption at $1675 \mathrm{~cm}^{-1}$ was attributable to a conjugated carbonyl group. The ${ }^{1} \mathrm{H}$ NMR spectrum (Table 1) showed no olefinic proton, whereas the ${ }^{13} \mathrm{C}$ NMR spec-

*Author to whom correspondence should be addressed. trum (Table 2) exhibited two olefinic carbons at $\delta 141.1$ and 143.7. An $\alpha$-hydroxy- $\alpha, \beta$-unsaturated ketone was inferred from these spectral data. An aromatic proton $\mathrm{H}$ 14 occurring at a low field $\delta 7.99$ (s) indicated 11 is an abietane-type diterpene having the carbonyl group at C7. The positions of $\mathrm{C}-5$ at $\delta 141.1$ and $\mathrm{C}-7$ at $\delta 179.7$ were deduced from a HMBC spectrum by correlation with C18 and $\mathrm{C}-14$, respectively. Compound 11 was assigned as 6,12-dihydroxyabieta-5,8,11,13-tetraen-7-one, an enol tautomer of 5-epixanthoperol (10). It was found that 10 yielded ca $50 \%$ of 11 on standing in $\mathrm{CDCl}_{3}$ for two months.

Compound $12\left(\mathrm{C}_{20} \mathrm{H}_{30} \mathrm{O}_{2}\right)$ showed the exact mass at $m / z$ 302.224. The structure was determined to be $6 \beta$ hydroxyferruginol by analysis of its NMR spectra (Tables 1 and 2). The compound has an equatorial $\mathrm{H}-6 x$, which exhibited small coupling constants with $\mathrm{C}-5$ and $\mathrm{C}-7$ protons $\left(J_{5,6}=2 \mathrm{~Hz}, J_{6,7 \beta}=6 \mathrm{~Hz}\right.$ and $\left.J_{6,7 \alpha}=3 \mathrm{~Hz}\right)$.

Compound $13\left(\mathrm{C}_{20} \mathrm{H}_{28} \mathrm{O}_{3}\right)$ showed the exact mass $\left[\mathrm{M}^{+}\right.$at $m / z$ 316.203. The ${ }^{13} \mathrm{C}$ NMR spectrum (Table 2) showed a signal at $\delta 187.1$ attributable to a carbonyl group and resonances at $\delta 126.2,138.6,149.5$ and 162.5 attributable to two $\mathrm{C}=\mathrm{C}$ double bonds. Taking these data and two alkenyl proton resonances at $\delta 6.03$ $(s)$ and $6.30(s)$, we considered 13 contained a cyclohexa-2,5-dienone moiety. Further evidence from the ${ }^{1} \mathrm{H}-{ }^{1} \mathrm{H}$ COSY, ${ }^{13} \mathrm{C}-{ }^{1} \mathrm{H}$ COSY, HMBC and NOE experiments support the structural assignment of 13 as $7 \alpha, 8 \alpha$-epoxy- $6 \alpha$-hydroxyabieta- $9(11), 13-d i e n-12$-one. The axial $\mathrm{H}-6 \beta$ (at $\delta 4.40$ ) appeared to couple with the axial $\mathrm{H}$ $5 \alpha(J=9 \mathrm{~Hz})$, but not with the equatorial H-7 $\beta$ (at $\delta 3.72$ ). Irradiation of $\mathrm{H}-7 \beta$ or Me- 10 caused 7.9 or $5.6 \%$ NOE of H- $6 \beta$, respectively, supporting the assigned stereochemistry. The HMBC spectrum revealed that $\mathrm{H}-11$ at $\delta 6.30$ was correlated with $\mathrm{C}-8, \mathrm{C}-10$ and $\mathrm{C}-13$, whereas $\mathrm{H}-14$ at $\delta 6.03$ was correlated with $\mathrm{C}-9$ and $\mathrm{C}-12$. Compound 13 
<smiles>[R]C1CCC2(C)c3cc(O)c(C(C)C)cc3CC([R])C2([C@@H]([R])C)C1(C)C</smiles>

$\begin{array}{ll}R^{1} & R^{2}\end{array}$

$1 \mathrm{H} \quad \mathrm{H}$

$7 \mathrm{OH} \mathrm{H}$

$12 \mathrm{H} \quad \mathrm{OH}$<smiles>CC(C)c1cc2c(cc1O)C1(C)CCCC(C)(C)C1C(=O)C2=O</smiles>

10<smiles>COc1cc2c(cc1C(C)C)C=C[C@@]1(C)[C@@H](C)CCC[C@]21C</smiles>

14

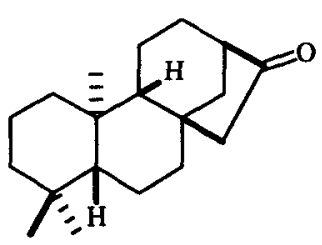

18<smiles>[R20]c1cc2c(cc1C(C)C)C=CC1C(C)(C)CCCC1(C)C2=C</smiles>

R

$3 \mathrm{Me}$<smiles>CC(C)c1cc2c(cc1O)[C@@]1(C)CCCC(C)(C)C1=C(O)C2=O</smiles>

11<smiles>[R2]c1cc([C@]2(C)CCCC(C)(C)[C@@]2([CH])C=O)c(C=O)cc1C(C)C</smiles>

R

$16 \mathrm{Me}$

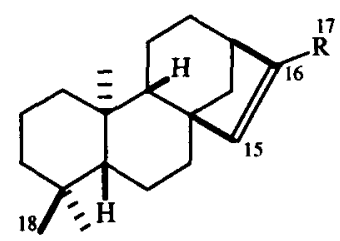

$R$

$19 \mathrm{CH}_{2} \mathrm{OH}$

20 CHO<smiles>[R4]c1c(C(C)C)cc2c(c1[R])C1(C)CCC([R])C(C)(C)[C@]1([C@@H]([R])C)C2=O</smiles>

$\begin{array}{llll}R^{1} & R^{2} & R^{3} & R^{4}\end{array}$

$\begin{array}{lllll}4 \mathrm{H} & \mathrm{H} & \mathrm{H} & \mathrm{H}\end{array}$

$\begin{array}{llll}5 \mathrm{H} & \mathrm{H} & \mathrm{H} & \mathrm{OH}\end{array}$

$6 \mathrm{H} \quad \mathrm{OH} \mathrm{H} \quad \mathrm{OH}$

$8 \mathrm{H} \quad \mathrm{H} \quad \mathrm{OH} \mathrm{OH}$

$9 \mathrm{H} \quad \mathrm{H} \quad \mathrm{OH} \mathrm{OMe}$<smiles>CC1=CC2=c3cc(C)c(C(C)C)cc3=CC(=O)[C@@H](O)C2=CC1=O</smiles>

13<smiles>CC(C)c1c(O)ccc2c1CC[C@@]1(C)[C@@H](C)CCC[C@@]21C</smiles>

17

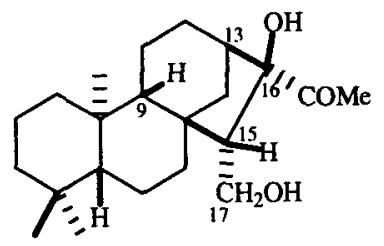

21 was subjected to acid-catalysed isomerization $(0.1 \mathrm{M} \mathrm{HCl}$, $\mathrm{MeOH}, \mathrm{CH}_{2} \mathrm{Cl}_{2}, 25^{\circ} \mathrm{C}, 16 \mathrm{hr}$ ) to give a main product 6 , presumably via opening of the epoxy ring to form an enol intermediate as shown in Scheme 1.

By analysis of the ${ }^{1} \mathrm{H}$ NMR spectrum, $14\left(\mathrm{C}_{21} \mathrm{H}_{30} \mathrm{O}\right)$ was assigned as $(5 R, 10 S)$-12-methoxyabieta-6,8,11,13tetraene, the C-5 epimer of 3 . The coupling constant of
$6 \mathrm{~Hz}$ between the equatorial H-5 $\beta$ and the vinyl H-6 in 14 is larger than the value of $2.5 \mathrm{~Hz}$ between the axial $\mathrm{H}-5 \alpha$ and H-6 in 3. On the other hand, 3 showed a W-shape coupling between $\mathrm{H}-5 \alpha$ and $\mathrm{H}-7(J=3 \mathrm{~Hz})$, whereas 14 lacked this phenomenon. The 5,10 -cis configuration was concluded by observing a $13.5 \%$ NOE of H-5 upon irradiation of the Me-10 group at $\delta 1.07$. The H-18 


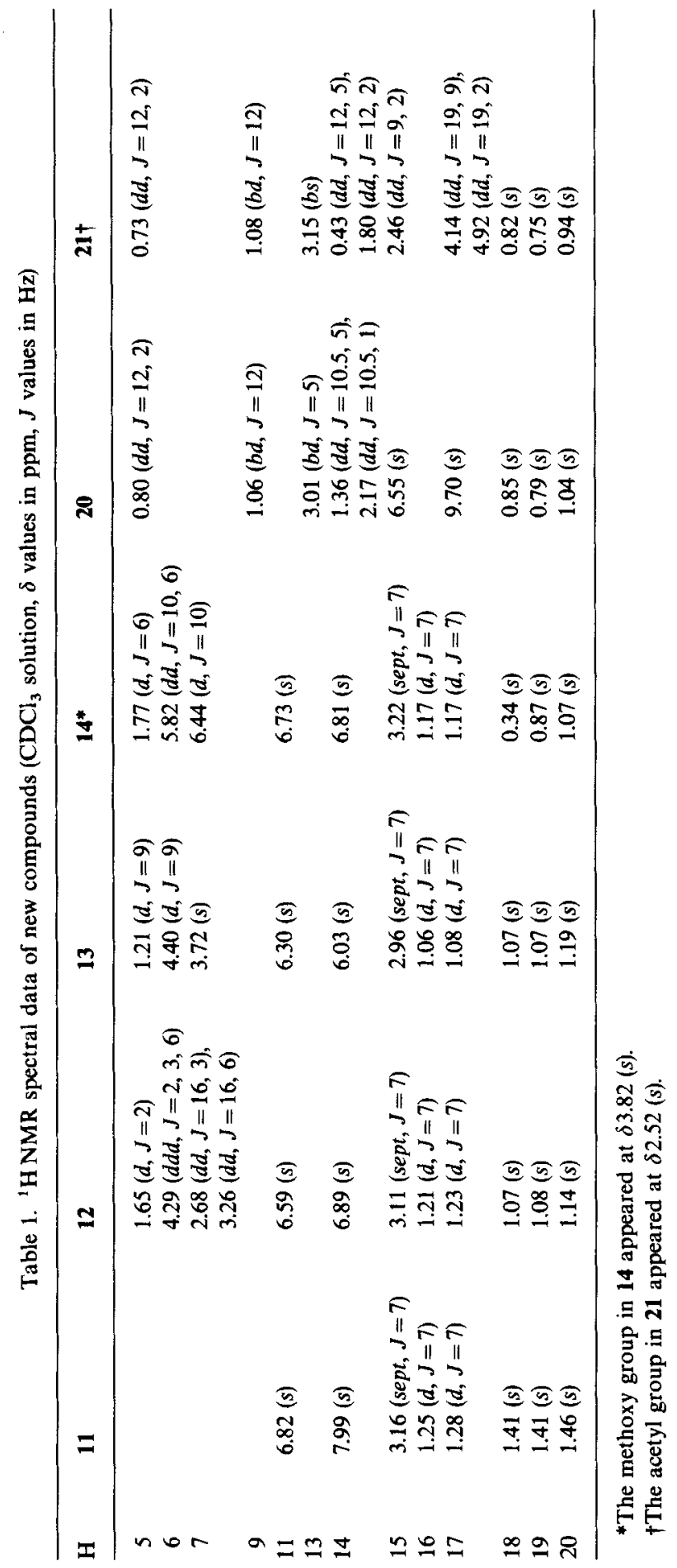


<smiles>CC(C)C1=CC2=CC(=O)[C@@H](O)[C@@]3(C)C(=O)C1=CC2(C)CCCC3(C)C</smiles>

13

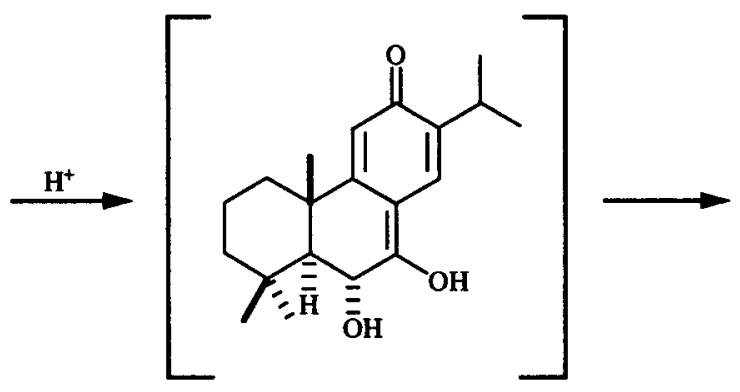

Scheme 1.

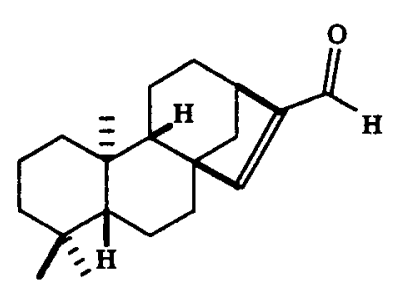

20

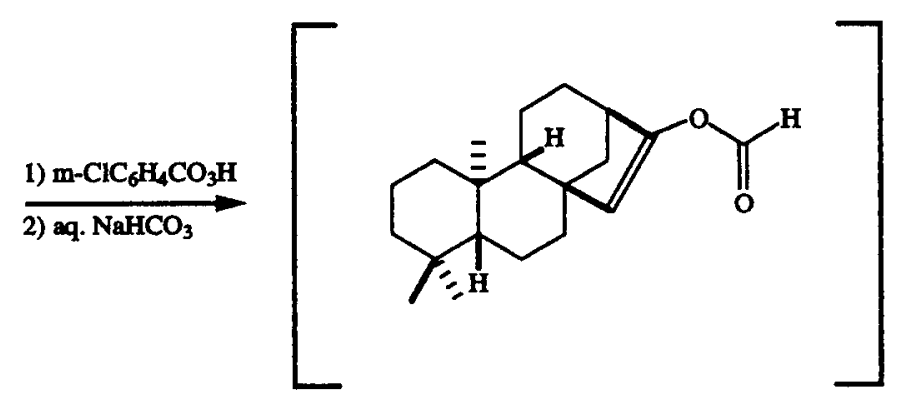

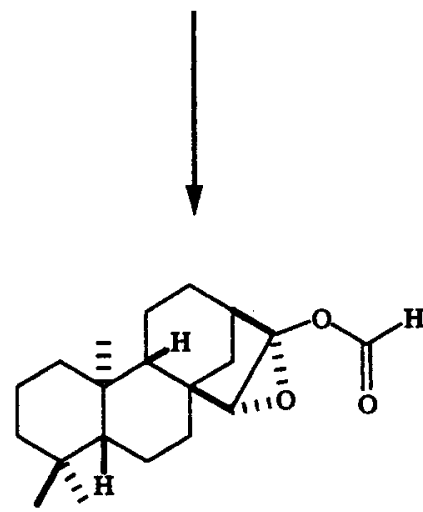

$\mathbf{A}$

Scheme 2.

resonance occurred at a remarkably high field, $\delta 0.34$, due to the shielding effect of the B-ring.

The known compounds ent-17-norkauran-16-one (18) [23] and ent-kaur-15-en-17-ol (19) [24] are laevorotatory. Compound 18 showed a carbon resonance at $\delta 222.8$ (C-16) attributable to a strained ketone. The new diterpene aldehyde $20\left(\mathrm{C}_{20} \mathrm{H}_{30} \mathrm{O}\right)$, ent-kaur-15-en-17-al, was correlated by oxidation of the alcohol 19 with manganese dioxide $\left(\mathrm{CH}_{2} \mathrm{Cl}_{2}, 20^{\circ}, 16 \mathrm{hr}\right)$. When 20 was subjected to oxidation with meta-chloroperbenzoic acid $\left(\mathrm{CH}_{2} \mathrm{Cl}_{2}, 0^{\circ}, 1 \mathrm{hr}\right)$ followed by treatment with aqueous sodium bicarbonate, the ketone 18 and an epoxyformate A were obtained (Scheme 2). The uncommon structure of
$21\left(\mathrm{C}_{22} \mathrm{H}_{36} \mathrm{O}_{3}\right),[\alpha]_{\mathrm{D}}^{25}+62.5^{\circ}$, was assigned as $(+)-16-$ acetylkaurane-16,17-diol. The acetyl group showed an IR absorption at $1700 \mathrm{~cm}^{-1}$ and a singlet at $\delta 2.52(\mathrm{~s})$ in the ${ }^{1} \mathrm{H}$ NMR spectrum. Assignments of proton and carbon resonances in 21 were assisted by ${ }^{13} \mathrm{C}-{ }^{1} \mathrm{H} C O S Y$ and $\mathrm{HMBC}$ spectra. The $\mathrm{H}-15$ and two geminal protons at $\mathrm{C}$ 17 appeared as an ABX pattern, at $\delta 2.46(d d, J=9,2 \mathrm{~Hz})$, $4.14(d d, J=19,9 \mathrm{~Hz})$ and $4.92(d d, J=19,2 \mathrm{~Hz})$. The stereochemistry was determined by NOE experiments, i.e. irradiation of the acetyl group at $\delta 2.52$ caused a $7.2 \%$ enhancement of $\mathrm{H}-13$ resonance at $\delta 3.15$ and irradiation of $\mathrm{H}-9$ at $\delta 1.08$ caused a $23.8 \%$ enhancement of $\mathrm{H}-15$ resonance. Since 18-21 were found in the same plant, the 
Table 2. ${ }^{13} \mathrm{CNMR}$ spectral data of new compounds $\left(\mathrm{CDCl}_{3}\right.$ solution, $\delta$ values in $\mathrm{ppm}^{*}$

\begin{tabular}{rrrrrrr}
\hline C & \multicolumn{1}{c}{11} & \multicolumn{1}{c}{12} & \multicolumn{1}{c}{13} & $14^{*}$ & \multicolumn{1}{c}{$\mathbf{2 0}$} & \multicolumn{1}{c}{$\mathbf{2 1 \dagger}$} \\
\hline 1 & 33.6 & 39.0 & 38.1 & 35.5 & 40.4 & 40.4 \\
2 & 17.6 & 19.0 & 18.1 & 19.3 & 18.5 & 18.5 \\
3 & 37.9 & 42.7 & 43.2 & 42.1 & 42.0 & 41.8 \\
4 & 35.9 & 34.1 & 34.7 & 35.1 & 33.3 & 33.1 \\
5 & 141.1 & 58.9 & 58.2 & 52.5 & 55.9 & 55.6 \\
6 & 143.7 & 68.6 & 66.4 & 125.9 & 18.7 & 19.8 \\
7 & 179.7 & 38.9 & 70.9 & 128.1 & 38.2 & 37.9 \\
8 & 120.9 & 123.5 & 56.2 & 127.4 & 51.0 & 46.0 \\
9 & 154.9 & 148.4 & 162.5 & 141.6 & 46.8 & 56.7 \\
10 & 40.3 & 38.1 & 38.0 & 37.0 & 39.8 & 39.5 \\
11 & 111.4 & 109.8 & 126.2 & 105.7 & 18.4 & 17.1 \\
12 & 157.7 & 151.3 & 187.1 & 156.4 & 25.3 & 26.4 \\
13 & 133.8 & 131.3 & 149.5 & 133.6 & 38.0 & 42.1 \\
14 & 125.6 & 126.6 & 138.6 & 124.7 & 43.0 & 36.1 \\
15 & 26.9 & 26.8 & 26.3 & 26.5 & 162.3 & 45.0 \\
16 & 22.3 & 22.2 & 21.3 & 22.7 & 148.5 & 117.7 \\
17 & 22.5 & 22.4 & 21.3 & 22.8 & 189.5 & 79.7 \\
18 & 28.2 & 34.6 & 35.3 & 20.6 & 33.5 & 33.5 \\
19 & 27.5 & 22.7 & 22.4 & 31.3 & 21.5 & 21.5 \\
20 & 35.1 & 22.7 & 21.6 & 33.4 & 17.7 & 17.4 \\
\hline
\end{tabular}

*The methoxy group in 14 appeared at $\delta 55.7$.

$\lceil$ The acetyl group in 21 appeared at $\delta 28.3$ and 201.8

dextrorotatory compound $\mathbf{2 1}$ was still considered an entkaurane derivative. However, it is unclear whether $\mathbf{2 1}$ is a natural product or an artefact.

In summary, we isolated totarol, 14 abietanes, two secoabietanes and four ent-kaurane derivatives from the leaves of $C$. japonica. Among the new compounds, the enol 11, epoxydienone 13, 5,10-cis abietane 14 and kauranediol had an additional acetyl group at $\mathrm{C}-16$ worth special note.

\section{EXPERIMENTAL}

General. Merck silica gel $60 \mathrm{~F}$ sheets were used for analytical TLC. HPLC was carried out on a Hibar Lichrosorb Si $60(7$ or $10 \mu \mathrm{m})$ column $(25 \times 1 \mathrm{~cm})$.

Plant material. The plant used in this study is introduced from Japan and cultivated in suburban Taipei. A voucher specimen has been deposited in our laboratory. The leaves $(1.4 \mathrm{~kg})$ of $C$. japonica $D$. Don. were exhaustively extracted with $\mathrm{Me}_{2} \mathrm{CO}$. The $\mathrm{Me}_{2} \mathrm{CO}$ extract was passed through a pad of charcoal, concd and re-extracted with EtOAc. The EtOAc-soluble portion ( $45 \mathrm{~g}$ ) was chromatographed on a silica gel column by elution with gradients of hexane and EtOAc. The appropriate frs were combined and purified by HPLC to give $14(10 \mathrm{mg}), 3$ (10 mg), 4 (5 mg), 17 (7 mg), 1 (35 mg), 20 (16 mg), 2 (5 mg), 16 (16 mg), 21 (11 mg), 12 (5 mg), 15 (18 mg), 13 (20 mg), 10 (5 mg), 11 (15 mg), 6 (12 mg), 9 (16 mg), 18 (6 mg), 19 $(27 \mathrm{mg}), 5(27 \mathrm{mg}), 8(10 \mathrm{mg})$ and $7(22 \mathrm{mg})$ in the order of increasing polarity.

Compounds 1-10, 15-19. Ferruginol (1): oily solid. $[\alpha]_{\mathrm{D}}^{20}+39.5^{\circ}\left(\mathrm{CHCl}_{3} ; c 1.3\right)$. 6,7-Dehydroferruginol (2): oil. $[\alpha]_{\mathrm{D}}^{25}-62^{\circ}\left(\mathrm{CHCl}_{3} ; c 0.5\right)$. 6,7-Dehydroferruginol methyl ether (3): oil. $[\alpha]_{\mathrm{D}}^{25}-87^{\circ}\left(\mathrm{CHCl}_{3} ; c 1\right) .{ }^{13} \mathrm{CNMR}$ $\left(\mathrm{CDCl}_{3}\right): \delta 19.1(\mathrm{C}-2), 20.1(\mathrm{C}-18), 22.5(\mathrm{C}-16,17), 22.9(\mathrm{C}-$ 19), 26.4 (C-15), 32.6 (C-20), 32.9 (C-4), 36.1 (C-1), 38.0 (C10), 41.1 (C-3), 51.1 (C-5), $55.6(\mathrm{MeO}), 104.8$ (C-11), 124.4 (C-14), 125.8 (C-8), 127.3 (C-6), 127.4 (C-7), 133.8 (C-13), 147.0 (C-9), 156.4 (C-12), 7-Dehydroabietanone (4): solid. $\mathrm{Mp} 85-87^{\circ} .[\alpha]_{\mathrm{D}}^{30}+13.5^{\circ}\left(\mathrm{CHCl}_{3} ; c 0.5\right) .{ }^{13} \mathrm{CNMR}$ $\left(\mathrm{CDCl}_{3}\right): \delta 18.8(\mathrm{C}-2), 21.2(\mathrm{C}-19), 23.3(\mathrm{C}-16), 23.7$ (C-17), 23.8 (C-20), 32.5 (C-18), 33.2 (C-4), 33.5 (C-15), 36.2 (C-6), 37.8 (C-10), 37.9 (C-1), 41.3 (C-3), 49.3 (C-5), 123.6 (C-12), 124.8 (C-11), 130.7 (C-8), 132.4 (C-14), 146.5 (C-13), 153.8 (C-9), 199.9 (C-7). Sugiol (5): solid. Mp 292-293.$[\alpha]_{\mathrm{D}}^{30}$ $+26.0^{\circ}$ (EtOH; c 1.5). $6 \alpha$-Hydroxysugiol (6): solid. $\mathrm{Mp} 207-208^{\circ} .[\alpha]_{\mathrm{D}}^{25}+35.3^{\circ}\left(\mathrm{CHCl}_{3} ; c 0.5\right) .{ }^{13} \mathrm{C} \mathrm{NMR}$ $\left(\mathrm{CDCl}_{3}\right): \delta 18.8$ (C-2), 21.8 (C-19), 22.2 (C-16), 22.4 (C-17), 24.7 (C-20), 26.8 (C-15), 34.1 (C-4), 35.8 (C-18), 38.8 (C-1), 39.2 (C-10), 42.9 (C-3), 55.9 (C-5), 73.9 (C-6), 110.2 (C-11), 121.8 (C-8), 127.2 (C-14), 133.4 (C-13), 156.5 (C-9), 158.9 (C-12), 199.7 (C-7). Hinokiol (7): solid. Mp 233-235. $[\alpha]_{\mathrm{D}}^{30}+74^{\circ}(\mathrm{EtOH} ; c 1.1) .{ }^{13} \mathrm{CNMR}$ (acetone- $\left.d_{6}\right): \delta 15.9$ (C-19), 19.7 (C-6), 22.7 (C-16), 22.9 (C-17), 25.1 (C-20), 27.2 (C-15), 28.5 (C-18), 28.8 (C-2), 30.7 (C-7), 37.8 (C-1), 37.9 (C-10), 39.5(C-4), 50.8 (C-5), 78.3 (C-3), 111.3 (C-11), 126.1 (C-8), 126.9 (C-14), 132.6 (C-13), 148.3 (C-9), 153.0 (C-12), 11-Hydroxysugiol (8): solid. Mp 192-193 ${ }^{\circ}[\alpha]_{\mathrm{D}}^{26}+21^{\circ}$ $\left(\mathrm{CHCl}_{3} ; c 1\right) .{ }^{13} \mathrm{C} \mathrm{NMR}\left(\mathrm{CDCl}_{3}\right): \delta 18.6(\mathrm{C}-20), 19.0(\mathrm{C}-2)$, 21.5 (C-19), 22.3 (C-16), 22.5 (C-17), 27.2 (C-15), 33.1 (C18), 33.4 (C-4), 35.5 (C-1), 36.7 (C-6), 40.1 (C-10), 41.0 (C3), 50.2 (C-5), 118.0 (C-14), 125.2 (C-8), 131.9 (C-13), 138.7 (C-9), 141.2 (C-11), 146.5 (C-12), 199.5 (C-7). Cryptojaponol (9): solid. Mp 203-204 $.[\alpha]_{\mathrm{D}}^{25}+25^{\circ}\left(\mathrm{CHCl}_{3} ; c\right.$ 1.6). ${ }^{13} \mathrm{C} \mathrm{NMR}\left(\mathrm{CDCl}_{3}\right): \delta 18.0(\mathrm{C}-2), 18.9(\mathrm{C}-20), 21.5(\mathrm{C}-19)$, 23.4 (C-16), 23.5 (C-17), 26.7 (C-15), 33.1 (C-18), 33.5 (C-4), 35.6 (C-6), 36.3 (C-1), 40.2 (C-10), 41.3 (C-3), 50.3 (C-5), 61.8 (MeO), 117.3 (C-14), 128.8 (C-8), 138.1 (C-11), 139.1 (C-13), 146.5 (C-9), 149.1 (C-12), 199.1 (C-7). 5-Epixanthoperol (10): solid. Mp 205-207 $7^{\circ}[\alpha]_{\mathrm{D}}^{25}+25^{\circ}\left(\mathrm{CHCl}_{3} ; c 0.5\right)$. 12-Hydroxy-6,7-secoabieta-8,11,13-triene-6,7-dial (15): solid. Mp 191-192 $.[\alpha]_{\mathrm{D}}^{30}+20^{\circ}\left(\mathrm{MeOH}_{3} ; c 1.2\right)$. 12Methoxy-6,7-secoabieta-8,11,13-triene-6,7-dial (16): oil. $[\alpha]_{\mathrm{D}}^{25}+51^{\circ}\left(\mathrm{CHCl}_{3} ; c 1\right) .{ }^{13} \mathrm{C} \mathrm{NMR}\left(\mathrm{CDCl}_{3}\right): \delta 19.5(\mathrm{C}-2)$, 22.3 (C-16, 17, 19), 23.4 (C-20), 26.5 (C-15), 30.6 (C-18), 32.2 (C-1), 33.6 (C-4), 37.6 (C-3), 40.9 (C-10), 55.4 (MeO), 64.9 (C-5), 109.2 (C-11), 127.6 (C-8), 133.2 (C-14), 135.0 (C13), 151.0 (C-9), 160.7 (C-12), 191.4 (C-7), 205.6 (C-6). Torarol (17): solid. Mp 125-126 ${ }^{\circ}[\alpha]_{\mathrm{D}}^{30}+40.2^{\circ}\left(\mathrm{EtOH}_{3}\right.$; c0.5). ent-17-Norkauran-16-one (18): solid. Mp 109-110 $[\alpha]_{\mathrm{D}}^{20}-20^{\circ}\left(\mathrm{CHCl}_{3} ; c 0.5\right) .{ }^{13} \mathrm{C} \mathrm{NMR}\left(\mathrm{CDCl}_{3}\right): \delta 18.0(\mathrm{C}-$ 20), 18.5 (C-11), 18.6 (C-2), 19.3 (C-6), 21.6 (C-19), 29.7 (C-12), 33.3 (C-4), 33.6 (C-18), 37.5 (C-7), 39.5 (C-10), 40.4 (C-1), 41.0(C-14), 41.9 (C-3), 42.5 (C-8), 48.0 (C-9), 55.0(C13), 55.2 (C-15), 56.1 (C-5), 222.8 (C-16). ent-Kaur-15-en17-ol (19): solid. Mp 85-86 $.[\alpha]_{\mathrm{D}}^{25}-33^{\circ}\left(\mathrm{CHCl}_{3} ; c 1\right)$. ${ }^{13} \mathrm{CNMR}\left(\mathrm{CDCl}_{3}\right): \delta 17.6(\mathrm{C}-20), 18.6(\mathrm{C}-2,11), 19.2(\mathrm{C}-6)$, 21.6 (C-19), 25.7 (C-12), 33.3 (C-4), 33.5 (C-18), 39.3 (C-7), 39.5 (C-10), 40.5 (C-1), 41.2 (C-13), 42.1 (C-3), 43.9 (C-14), 48.4 (C-9), 49.0 (C-8), 56.0 (C-5), 61.3 (C-17), 136.1 (C-15), 146.0 (C-16).

6,12-Dihydroxyabieta-5,8,11,13-tetraen-7-one (11). Needles from $\mathrm{CHCl}_{3} \cdot \mathrm{Mp} 189-190^{\circ} \cdot[\alpha]_{\mathrm{D}}^{26}-8.5^{\circ}\left(\mathrm{CHCl}_{3}\right.$; 
c0.9). TLC (20\% EtOAc in hexane) $R_{f}$ 0.55. IR $v_{\max }^{\mathrm{KBr}} \mathrm{cm}^{-1}: 3326,1675,1601,1502,1380$. UV $\lambda_{\max }^{\mathrm{MeOH}} \mathrm{nm}(\varepsilon)$ : 335 (7900), 309 (6140), 281 (7480), 261 (3510), 250 (6270), 236 (4540). EIMS (70 eV) $m / z$ rel. int.: 314 [M] $^{+}(100), 299$ (16), 271 (42), 245 (75), 229 (30), 215 (26), 203 (20). HRMS for $\mathrm{C}_{20} \mathrm{H}_{26} \mathrm{O}_{3}$ requires: 314.1883. Found: 314.1881 . $6 \beta$-Hydroxyferruginol (12). Oily solid. $[\alpha]_{\mathrm{D}}^{20}+9.4^{\circ}$ $\left(\mathrm{CHCl}_{3} ; c 0.5\right)$. TLC ( $\mathrm{CHCl}_{3}$-hexane-EtOAc, 10:9:1) $\boldsymbol{R}_{\boldsymbol{f}}$ 0.33. IR $v_{\max }^{\text {Neat }} \mathrm{cm}^{-1}: 3378,1613,1499,1373$. EIMS $(70 \mathrm{eV})$ $m / z$ rel. int:: 302 [M] $^{+}(100), 287(24), 269(81), 227(53)$, 199 (31), $157(27), 149(19)$. HRMS for $\mathrm{C}_{20} \mathrm{H}_{30} \mathrm{O}_{2}$ requires: 302.2247. Found: 302.2242.

$7 \alpha, 8 \alpha$-Epoxy-6 $\alpha$-hydroxyabieta-9(11),13-dien-12-one (13). Oil. $[\alpha]_{\mathrm{D}}^{25}-42.5^{\circ}\left(\mathrm{CHCl}_{3} ; c 2\right)$. TLC (13\% EtOAc in hexane) $R_{f} 0.32$. IR $v_{\max }^{\text {Neat }} \mathrm{cm}^{-1}: 3411,1649,1614,1379$, 1232, 921. UV $\lambda_{\max }^{\mathrm{CHCl}_{3}} \mathrm{~nm}(\varepsilon): 261$ (9300), 250 (7900). EIMS $(70 \mathrm{eV}) \mathrm{m} / \mathrm{z}$ rel. int.: $316[\mathrm{M}]^{+}(63), 301(40), 287(80), 273$ (20), 231 (55), 203 (100), 163 (52). HRMS for $\mathrm{C}_{20} \mathrm{H}_{28} \mathrm{O}_{3}$ requires: 316.2039 . Found: 316.2031.

(5R,10S)-12-Methoxyabieta-6,8,11,13-tetraene (14). Oil. $[\alpha]_{\mathrm{D}}^{28}-266^{\circ}\left(\mathrm{CHCl}_{3} ; c 1\right)$. TLC (hexane) $R_{f} 0.56$. IR $v_{\max }^{\text {Neat }} \mathrm{cm}^{-1}: 3022,1601,1493,1380,1362,1246$. UV $\lambda_{\max }^{\mathrm{MeOH}} \mathrm{nm}(\varepsilon): 281$ (8900), 245 (1900), 222 (25 600). EIMS $(70 \mathrm{eV}) \mathrm{m} / \mathrm{z}$ rel. int.: 298 [M] $^{+}(49), 283$ (15), 241 (9), 227 (22), 216 (100), 199 (35), 173 (38). HRMS for $\mathrm{C}_{21} \mathrm{H}_{30} \mathrm{O}$ requires: 298.2298. Found: 298.2296.

ent-Kaur-15-en-17-al (20). Crystals from EtOAchexane (6:94). Mp 115-116 $.[\alpha]_{\mathrm{D}}^{25}-99^{\circ}\left(\mathrm{CHCl}_{3}\right.$; $c 1)$. TLC (6\% EtOAc in hexane) $R_{f} 0.45$. IR $v_{\max }^{\mathbf{K B r}}$ $\mathrm{cm}^{-1}: 2795,1660,1596$. UV $\lambda_{\max }^{\mathrm{MeOH}} \mathrm{nm}(\varepsilon): 248$ (7700), 217 (940). EIMS $(70 \mathrm{eV}) \mathrm{m} / \mathrm{z}$ rel. int.: $286 \mathrm{[M}^{+}(80)$, 271 (65), 253 (22), 175 (37), 123 (71), 109 (45), 91 (100). HRMS for $\mathrm{C}_{20} \mathrm{H}_{30} \mathrm{O}$ requires: 286.2298. Found: 286.2304. A soln of $20(25 \mathrm{mg}$, obtained from oxidation of 19 with $\left.\mathrm{MnO}_{2}\right)$ in $\mathrm{CH}_{2} \mathrm{Cl}_{2}\left(5 \mathrm{~cm}^{3}\right)$ was stirred with $m$ CPBA (1.2 equiv) at $0^{\circ}$ for $1 \mathrm{hr}$ and treated subsequently with $0.5 \mathrm{M}$ aq. $\mathrm{NaHCO}_{3}(3 \mathrm{ml})$ for $1 \mathrm{hr}$. The organic phase was concd and sepd by HPLC with elution of EtOAc-hexane (4.5:95.5) to give the ketone $18(2.5 \mathrm{mg})$ and the epoxyformate $\mathrm{A}(5 \mathrm{mg})$ accompanied by recovery of $20(15 \mathrm{mg})$. A: solid. Mp 78-79 $.[\alpha]_{\mathrm{D}}^{25}-5^{\circ}\left(\mathrm{CHCl}_{3}\right.$; $c 0.5)$. TLC (4.5\% EtOAc in hexane) $R_{f} 0.42$. IR $v_{\max }^{\mathbf{K B r}} \mathrm{cm}^{-1}: 1745,1182,971$. EIMS $(70 \mathrm{eV}) \mathrm{m} / z$ rel. int.: 318 $[\mathrm{M}]^{+}(5), 303(9), 290(70), 275(40), 257(25), 247(30), 229$ (25), $123(100) .{ }^{1} \mathrm{H} \mathrm{NMR}\left(\mathrm{CDCl}_{3}\right): \delta 0.76(d d, J=12,2 \mathrm{~Hz}$, H-5), 0.78 (s, H-19), 0.85 (s, H-18), 0.99 (s, H-20), 1.08 (bd, J $=12 \mathrm{~Hz}, \mathrm{H}-9), 2.75$ (bd, $J=5 \mathrm{~Hz}, \mathrm{H}-13), 3.20$ (s, H-15), $8.10\left(s, \mathrm{HCO}_{2}\right) \cdot{ }^{13} \mathrm{C} \mathrm{NMR}\left(\mathrm{CDCl}_{3}\right): \delta 17.5(\mathrm{C}-20), 18.1(\mathrm{C}-$ 11), 18.4 (C-2, 6), 21.5 (C-19), 25.6 (C-12), 32.8 (C-7), 33.3 (C-4), 33.5 (C-18), 34.9 (C-14), 37.1 (C-13), 39.1 (C-10), 40.4 (C-1), 41.9 (C-3), 42.5 (C-8), 50.4 (C-9), 55.9 (C-5), 65.3 (C15), 86.6 (C-16), $159.5\left(\mathrm{HCO}_{2}\right)$. HRMS for $\mathrm{C}_{20} \mathrm{H}_{30} \mathrm{O}_{3}$ requires: 318.2196 . Found: 318.2190 .

(+)-16-Acetylkaurane-16,17-diol (21). Crystals from hexane. Mp 170-171 ${ }^{\circ} .[\alpha]_{\mathrm{D}}^{25}+62.5^{\circ}\left(\mathrm{CHCl}_{3} ; c 0.9\right)$. TLC (10\% EtOAc in hexane) $R_{f} 0.40$. IR $v_{\max }^{\mathrm{KBr}} \mathrm{cm}^{-1}: 3450$, 3380,1700 . EIMS (70 eV) $\mathrm{m} / z$ rel. int.: $348[\mathrm{M}]^{+}(2), 330$ (8), 314 (30), 299 (40), $271(28), 217(25), 177(57), 163(100)$.
HRMS for $\mathrm{C}_{22} \mathrm{H}_{36} \mathrm{O}_{3}$ requires: 348.2666. Found: 348.2658.

Acknowledgement-We are grateful to the National Science Council for financial support.

\section{REFERENCES}

1. Gan, W. S. (1958) Manual of Medicinal Plants in Taiwan Vol. 1, pp. 54-55. Nat. Res. Inst. Chin. Med., Taipei.

2. Miura, H., Kawano, N. and Waiss, Jr A. C. (1966) Chem. Pharm. Bull. 14, 1404.

3. Appleton, R. A., McCrindle, R. and Overton, K. H. (1968) Phytochemistry 7, 135.

4. Appleton, R. A., McCrindle, R. and Overton, K. H. (1970) Phytochemistry 9, 581.

5. MacMillan, J. and Walker, E. R. H. (1972) J. Chem. Soc. Perkin Trans. I 981.

6. Yasue, M., Ogiyama, K. and Saitoh, M. (1976) J. Jap. Forest Soc. 58, 285.

7. Yasue, M., Ogiyama, K., Ebishigawa, S., Kondo, K. and Nishina, K. (1978) J. Jap. Forest Soc. 60, 345.

8. Shieh, B., Iizuka, Y. and Matsubara, Y. (1981) Agric. Biol. Chem. 45, 1493.

9. Yatagai, M. and Sato, T. (1986) Biochem. Syst. Ecol. 14, 469.

10. Matsui, T., Ohishi, H. and Nakayama, N. (1989) Kogakubu Kenkyu Hokoku 35, 157.

11. Vernin, G., Faure, R. and Pieribattesti, J. C. (1990) J. Essent. Oil Res. 2, 211.

12. Su, W.-C., Fang, J.-M. and Cheng, Y.-S. (1993) Phytochemistry 34, 779.

13. Bredenberg, J. B-Son (1957) Acta Chem. Scnad. 11, 932.

14. Lin, Y.-T., Kuo, Y.-H. and Chang, B.-H. (1975) J. Chin. Chem. Soc. 22, 331.

15. Defaye-Duchateau, G. (1964) Bull. Soc. Chim. Fr. 1469.

16. Kupchan, S. M., Karim, A. and Marcks, C. (1969) J. Org. Chem. 34, 3912.

17. Fang, J.-M., Sheu, C.-M. and Cheng, Y.-S. (1986) J. Chin. Chem. Soc. 33, 245.

18. Hueso-Rodriguez, J. A., Jimeno, M. L., Rodriguez, B., Savona, G. and Bruno, M. (1983) Phytochemistry 22, 2005.

19. Matsumoto, T., Ohsuga, Y., Harada, S. and Fukui, K. (1977) Bull. Chem. Soc. Jpn. 50, 266.

20. Bredenberg, J. B-Son (1960) Acta Chem. Scand. 14, 385.

21. Fang, J.-M., Jan, S.-T. and Cheng, Y.-S. (1986) J. Chem. Research (S) 350.

22. Ying, B. P. and Kubo, I. (1991) Phytochemistry 30, 1951.

23. Cross, B. E., Hanson, J. R., Cambie, R. C., Briggs, L. H. and Rutledge, P. S. (1963) Proc. Chem. Soc. 17.

24. Bohlmann, F., Kramp, W., Jakupovic, J., Robinson, H. and King, R. M. (1982) Phytochemistry 21, 399. 